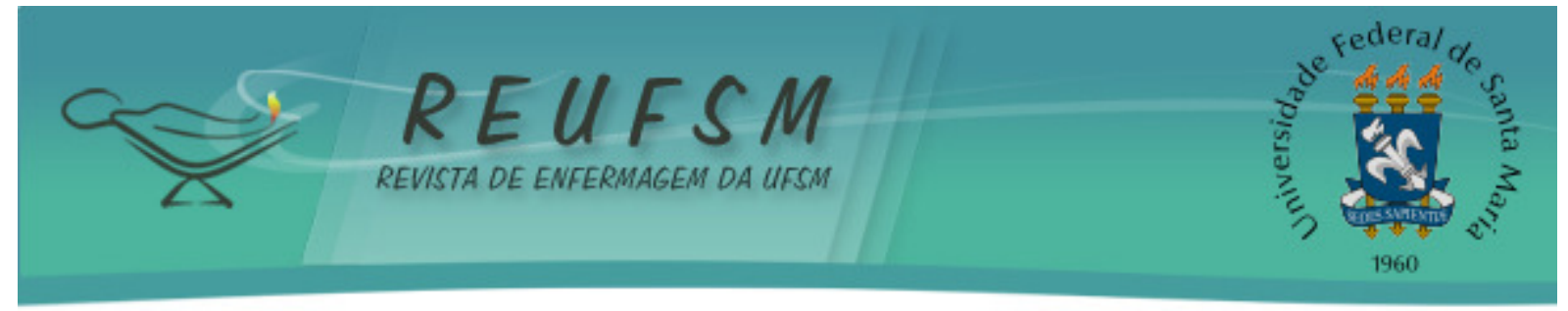

ARTIGO ORGINAL

\title{
CUIDADOS PALIATIVOS NA UTI: COMPREENSÃO, LIMITES E POSSIBILIDADES POR ENFERMEIROS
}

\section{PALLIATIVE CARE IN ICU: COMPREHENSION, LIMITS AND POSSIBILITIES FOR NURSES CUIDADOS PALIATIVOS EN LA UCI: LA COMPRENSIÓN, LIMITES Y POSIBILIDADES PARA LOS ENFERMEROS}

\author{
Nara Calazans Balbino Barros ${ }^{1}$ \\ Cecília Danielle Bezerra Oliveira ${ }^{2}$ \\ Estela Rodrigues Paiva Alves ${ }^{3}$ \\ Inácia Sátiro Xavier de França ${ }^{4}$ \\ Raquel Medeiros Nascimento ${ }^{5}$ \\ Maria Eliane Moreira Freire ${ }^{6}$
}

RESUMO: Objetivo: verificar a compreensão, limites e possibilidades enfrentadas por enfermeiros para realizar cuidados paliativos aos pacientes na UTI de um hospital de João Pessoa/PB. Método: estudo qualitativo realizado em abril e maio de 2011 com seis enfermeiras da UTI. Utilizou-se um questionário contendo dados de caracterização da amostra e duas questões norteadoras. As informações foram analisadas conforme o Discurso Sujeito Coletivo. Resultados: ideias centrais: ações multiprofissionais que visam promover conforto e bem-estar aos pacientes e seus familiares; ações que não resultam em melhora. Dos limites: ausência de profissionais preparados e de protocolo específico para cuidados paliativos; apoio espiritual ao paciente, necessidade de prescrição médica e não oferta de cuidados paliativos. Das possibilidades: realizar ações que proporcionam conforto e alívio da dor. Conclusão: os enfermeiros da UTI apresentam limitações com relação à assistência ao paciente terminal e sua família.

Descritores: Enfermagem; Unidade de terapia intensiva; Cuidados paliativos.

ABSTRACT: Objective: to verify the comprehension, limits and possibilities faced by nurses to perform palliative care for patients in the ICU of a hospital in João Pessoa/PB. Method: qualitative study conducted in April and May 2011 with six ICU nurses. We used a questionnaire containing data characterizing the sample and two guiding questions. The data were analyzed according to the Collective Subject Discourse. Result: multidisciplinary actions to promote well-being and comfort to patients and their families; actions that do not result in improvement. Limits: ack of trained professionals and a specific protocol for palliative care; spiritual support to the patient, a prescription and do not offer palliative care. The possibilities: perform actions that provide comfort and pain relief. Conclusion: ICU nurses have limitations with respect to patient care terminal and your family.

Descriptors: Nursing; Intensive care units; Hospice care.

\footnotetext{
${ }^{1}$ Enfermeira. Especialista em Terapia Intensiva. Faculdade Santa Emília de Rodat - FASER. Email: naracalazans@hotmail.com

${ }^{2}$ Enfermeira. Mestre em Enfermagem. Universidade Estadual da Paraíba - UEPB. Email: cecilia.dani@hotmail.com

${ }^{3}$ Enfermeira. Mestre em Enfermagem. Univesidade Federal de Pernambuco - UFPE. Email: rodrigues.estela@gmail.com

${ }^{4}$ Graduanda em Enfermagem. Faculdade Maurício de Nassau. Email: raquelmedeiros05@hotmail.com

${ }^{5}$ Enfermeira. Doutora em Enfermagem. Universidade Estadual da Paraíba - UEPB. Email: inacia.satiro@gmail.com

${ }^{6}$ Enfermeira. Mestre em Enfermagem. Universidade Federal da Paraíba - UFPB. Email: enf.elimoreirafreire@gmail.com
} 


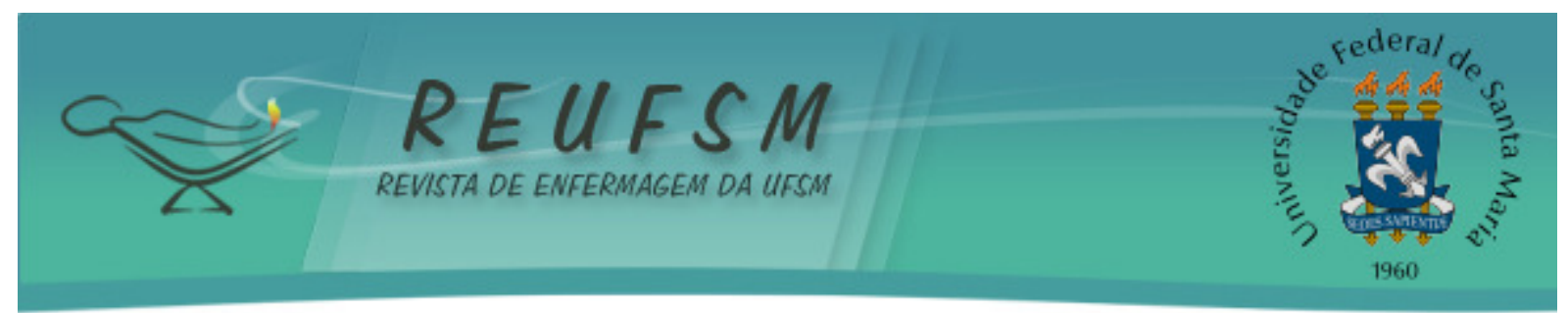

RESUMEN: Objetivo: comprobar la comprensión, límites y posibilidades enfrentadas por los enfermeros para realizar los cuidados paliativos a los pacientes en la $\mathrm{UCl}$ de un hospital en João Pessoa/PB. Método: estudio cualitativo realizado en abril y mayo de 2011 con seis enfermeras de $\mathrm{UCl}$. Se utilizó un cuestionario con los datos que caracterizan la muestra y dos preguntas orientadoras. Los datos fueron analizados de acuerdo con el Discurso del Sujeto Colectivo. Resultado: ideas centrales: acciones multiprofesionales para promover la comodidad y el bienestar de los pacientes y sus familias, acciones que no conducen a una mejora. Límites: la falta de profesionales capacitados y un protocolo específico de cuidados paliativos, apoyo espiritual al paciente, la prescripción y la no prestación de los cuidados paliativos. Posibilidades: tomar acciones que proporcionan comodidad y alivio del dolor. Conclusión: enfermeros de la $\mathrm{UCl}$ tienen limitaciones con relación a la atención al paciente terminal y su familia.

Descriptores: Enfermería; Unidades de cuidados intensivos; Cuidados paliativos.

\section{INTRODUÇÃO}

A Organização Mundial da Saúde (OMS) conceitua cuidados paliativos como uma abordagem que visa melhorar a qualidade de vida dos pacientes e de suas famílias ao enfrentar problemas associados a doenças terminais. Essa abordagem é realizada por meio da identificação precoce, avaliação religiosa e alívio da dor com base nos aspectos de ordem física, espiritual e psicossocial. ${ }^{1}$

O cuidado paliativo preocupa-se com as necessidades do paciente e não com o seu diagnóstico. São cuidados integrais aos pacientes e familiares, realizados por profissionais membros de uma equipe multidisciplinar, todos com sua importância, já que tal cuidado planeja diminuir o sofrimento humano. ${ }^{2-3}$

Os cuidados prestados à pacientes sem possibilidades de cura terapêutica integram uma proposta de assistência humanizada. Neste sentido, o paciente deverá ter sua dor amenizada, seu bem-estar priorizado e suas crenças consideradas, para que ele possa aceitar sua condição como um processo natural da finitude. Para tanto, é indispensável que todas as ações terapêuticas sejam planejadas com a participação do paciente, família e da equipe de saúde. ${ }^{4}$

Nesta proposta, a assistência de enfermagem é fundamental para o sucesso das intervenções prestadas, pois os cuidados devem ser integrais, centrados nas necessidades do paciente, com ações efetivas, respeitando a autonomia do paciente. Porém, é evidente a carência de unidades hospitalares que possibilitem a oferta desse cuidado prestado de forma individualizada, bem como uma notória deficiência no ensino ao tratar-se da atuação do enfermeiro diante da temática "paciente terminal". ${ }^{4}$

Com o incremento das tecnologias na área da saúde, ao longo dos tempos, a morte do indivíduo passou do ambiente domiciliar para hospitalar com o intuito de tentar evitar a morte e promover maior tempo de sobrevida. No âmbito hospitalar é na Unidade de Terapia Intensiva - UTI, que se concentra o maior número de pacientes críticos e dentre estes há pacientes com situações clínicas reversíveis, como também pacientes com doenças crônicas, sem perspectiva terapêutica de cura e que evoluem para estágio de terminalidade. ${ }^{4}$

No contexto da UTI, a luta pela vida de pacientes criticamente enfermos tem sido permeada por técnicas e aparatos tecnológicos especializados, porém diante da terminalidade do ser há evidências do despreparo de profissionais ao implementar ações que se sobrepõe ao conforto do paciente que está morrendo, com condutas fúteis que só lhe causam dor e prolongamento do sofrimento. ${ }^{4-5}$

A formação acadêmica do profissional de saúde não contempla abordagens teóricas mais profundas e direcionadas para a morte e o morrer, havendo poucas discussões sobre a temática entre os graduandos; e, ao se defrontar com a execução de 


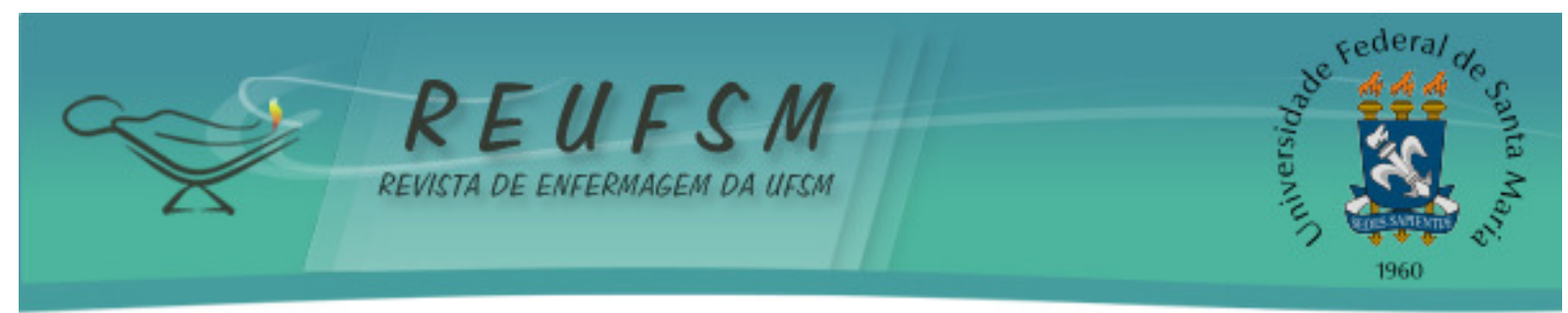

ações intensivistas, parece que ações paliativas não são apropriadas para este espaço assistencial, onde a concepção de cuidados paliativos ainda se apresenta distante da filosofia de atendimento da unidade de cuidados intensivos.

No Brasil, vários estudos têm alertado para a importância da realização dos cuidados paliativos, garantindo a qualidade de vida, aliviando a dor e priorizando sempre o interesse do paciente. $O$ início precoce dos cuidados paliativos pode proporcionar melhor adaptação do paciente e seus familiares com a situação, com fortalecimento de estratégias de enfrentamento, estabelecendo-se um cuidado individualizado e humanizado. . $^{5}$

A enfermagem em sua prática assistencial tem se destacado como um elo importante entre o paciente, os demais profissionais e os familiares; assim a compreensão deste profissional sobre as modalidades de cuidados paliativos é fundamental para sua inserção no planejamento, direcionamento e execução de ações paliativas no contexto da terapia intensiva.

Levando em consideração a importância do profissional de enfermagem nesses cuidados, abre-se uma discussão para responder aos seguintes questionamentos: qual a compreensão dos enfermeiros que atuam na UTI sobre cuidados paliativos? Que limites e possibilidades os enfermeiros enfrentam para realizar os cuidados paliativos nesse contexto?

Diante do exposto, o presente estudo teve como objetivos verificar a compreensão, os limites e as possibilidades enfrentadas por enfermeiros para realizar cuidados paliativos aos pacientes na UTI de um hospital público de João Pessoa/PB.

\section{MÉTODO}

Estudo exploratório-descritivo com abordagem qualitativa, realizado em uma UTI de um hospital de João Pessoa/PB, envolvendo seis enfermeiros integrantes do quadro de pessoal do setor da instituição, que aceitaram participar livremente da pesquisa. A escolha do local se deu pela grande concentração da população alvo, viabilidade da investigação e por fim, acessibilidade.

Os critérios para composição da amostra foram: tempo e experiência maior que um ano em UTI e disponibilidade para participar da pesquisa.

Por se tratar de um estudo qualitativo, a amostra foi alcançada conforme a saturação dos dados ${ }^{7}$, portanto, não sendo uma condição, a delimitação do número de participantes. A coleta foi realizada durante os meses de abril e maio de 2011.

Para a coleta dos dados foi utilizado um questionário semiestruturado, contendo questões de caracterização da amostra (idade, sexo, estado civil e tempo de trabalho na área) e duas questões norteadoras elaboradas pelos pesquisadores: 1) Qual a sua compreensão sobre cuidados paliativos? 2) Que limites e possibilidades você encontra para realizar os cuidados paliativos?

Os dados foram coletados após todos os participantes assinarem o Termo de Consentimento Livre e Esclarecido, antecedidos de esclarecimentos sobre os propósitos da pesquisa, riscos e benefícios, processo de coleta dos dados e apresentação da carta de autorização para o desenvolvimento da pesquisa à coordenadora e aos enfermeiros da UTI.

A entrega dos questionários ocorreu respeitando a jornada de trabalho de cada enfermeiro, bem como o seu recolhimento, os quais foram respondidos por escrito e sem a presença da autora resguardando a privacidade e anonimato dos atores. Sob esse aspecto, optou-se por denominar os participantes de Enf.1, Enf.2,..., Enf.6 quando revelados seus respectivos depoimentos no Discurso do Sujeito Coletivo.

Os dados foram analisados com base no referencial teórico de Lefévre ${ }^{8}$ dispostos de acordo com a técnica do Discurso do Sujeito Coletivo (DSC). Este método tem como proposta contemplar o pensamento, o sentido e o posicionamento de pessoas sobre determinados assuntos, revelados durante entrevistas e consolidados em forma de discurso coletivo; para produção do DSC são utilizadas quatro figuras metodológicas: ExpressãoChave $(\mathrm{ECH})$ formada por trechos dos depoimentos que revelem a essência do conteúdo das 


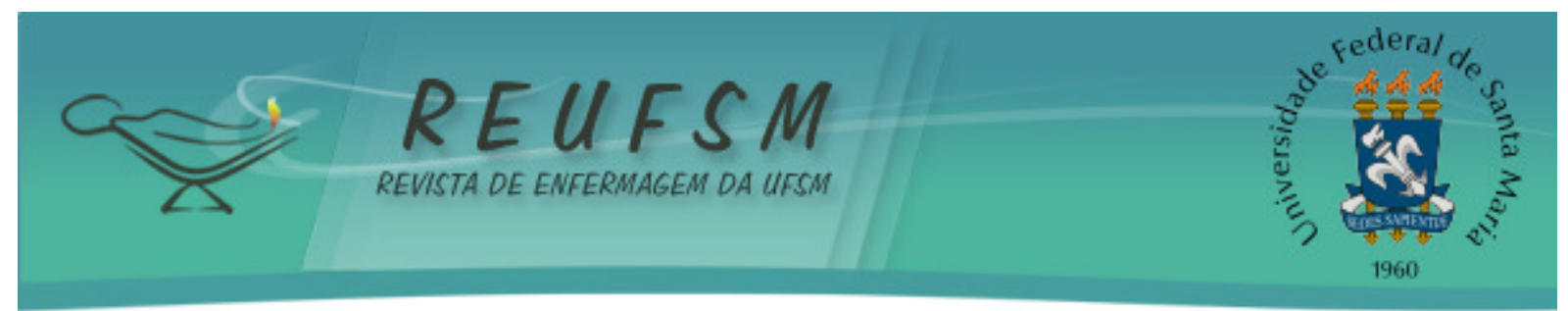

representações; Ideia Central (IC) que é a expressão linguística que descreve o sentido presente nos depoimentos; Ancoragem (AC) é uma afirmação redigida de forma positiva e que represente a ideia básica que sustenta o discurso, ou seja, revela a Representação Social daquilo que está sob enfoque; DSC é a reunião dos discursos, redigidos na primeira pessoa do singular, que apresentam ideias centrais ou ancoragens semelhantes.

Para a operacionalização desta técnica faz-se necessário os seguintes passos: seleção das ECH; apreensão da IC e construção do DSC de cada uma das ECH; identificação das Ideias centrais semelhantes ou complementares, transcrevendo-se na íntegra os termos utilizados pelo participante do estudo e a reunião das expressões chave referente às ideias centrais em um discurso síntese, constituindo assim, o DSC. ${ }^{8}$

0 estudo considerou todos os aspectos éticos em pesquisas que envolvem seres humanos, no que diz respeito à privacidade, ao anonimato e ao direito de desistir da pesquisa em qualquer momento, sem prejuízo para os participantes do estudo, normatizados pela Resolução 196/96 do Conselho Nacional de Saúde. ${ }^{9}$ A pesquisa foi aprovada pelo Comitê de Ética e Pesquisa da Faculdade de Enfermagem Santa Emília de Rodat, sob o protocolo n 014/2011.

\section{RESULTADOS E DISCUSSÕES}

\section{Caracterização dos sujeitos entrevistados}

A amostra foi constituída por seis enfermeiros que atuam na UTI da instituição selecionada para o estudo. De acordo com a caracterização sociodemográfica dos participantes, destaca-se que três tinham idade entre 30 e 39 anos e três entre 40 e 49 anos, cinco eram do sexo feminino e um do sexo masculino, cinco referiram estar solteiros e um casado, destes três são especialistas em terapia intensiva. 0 tempo de atuação como enfermeiro variou entre os participantes de cinco a 28 anos de trabalho, com carga horária semanal variando de 24 a 60 horas semanais.

\section{Análise dos Discursos do Sujeito Coletivo (DSC)}

Conforme os objetivos propostos neste estudo, a pergunta inicial dirigida aos enfermeiros participantes, buscou a compreensão destes sobre cuidados paliativos. Os depoimentos individuais constituíram os discursos, de onde emergiram duas ideias centrais: (1) ações multiprofissionais que visam promover conforto e bem estar aos pacientes e seus familiares; (2) ações que não resultam em melhora.

\begin{tabular}{|l|l|}
\hline Ideia central (1) & DSC (1) \\
\hline $\begin{array}{l}\text { Ações multiprofissionais que visam } \\
\text { promover conforto e bem-estar aos } \\
\text { pacientes e seus familiares. }\end{array}$ & $\begin{array}{l}\text { f... é a prática do atendimento, envolvendo os aspectos } \\
\text { fisicos, emocionais, sociais [...] (Enf.: 1); É um conjunto } \\
\text { de ações multiprofissionais que visam oferecer conforto } \\
\text { ao paciente e seus familiares [...]. (Enf.: 2); São cuidados } \\
\text { ao bem-estar do paciente, e que nós como enfermeiros } \\
\text { fazemos sem autorização médica [...]. (Enf.: 3); "Dar a } \\
\text { assistência precisa ao paciente que tem doença crônica e } \\
\text { dor... aquela definitivamente acentuada" (Enf.: 4). }\end{array}$ \\
\hline DSC (2) \\
\hline Idéia central (2) & $\begin{array}{l}\text { DSC: [...] São os primeiros atendimentos admissionais [...] } \\
\text { (Enf.: 3, Enf.: 5); são aqueles cuidados prestados ao paciente } \\
\text { enfermo, mas que não resultarão em melhora [...]. (Enf.: 6). }\end{array}$ \\
\hline
\end{tabular}

Figura 1- Ideia Central, DSC dos Enfermeiros em resposta a questão: Qual a sua compreensão sobre cuidados paliativos? Fonte: Dados da pesquisa, 2011. 


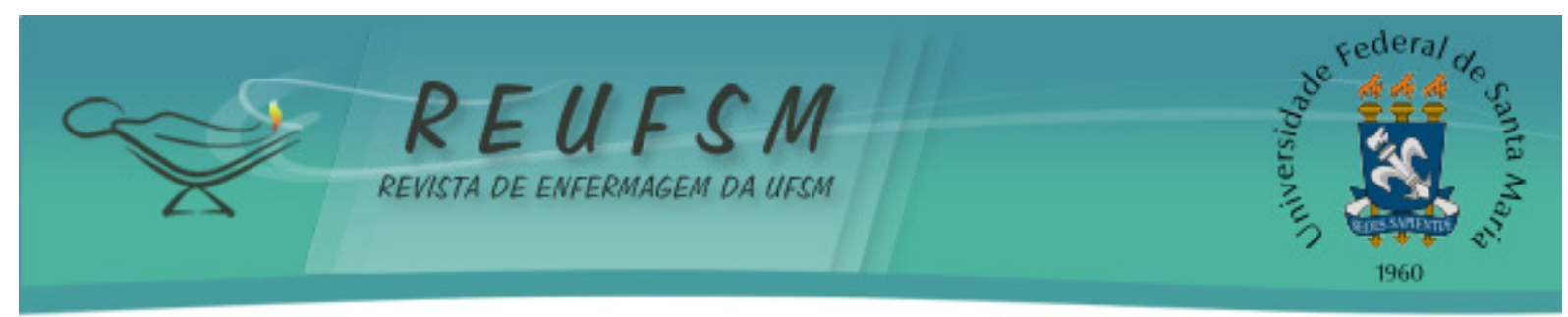

A metodologia utilizada permitiu que os enfermeiros, quando questionados, pudessem expressar o que eles entendiam sobre cuidados paliativos. A ideia central (1) que tem como significado "ações multiprofissionais que visam promover conforto e bem-estar aos pacientes e seus familiares" representa a essência do discurso dos enfermeiros com relação à compreensão dos mesmos sobre cuidados paliativos.

A definição de cuidados paliativos, conforme a OMS, integra alívio dos sintomas, da dor e do sofrimento de pacientes que sofrem de doenças crônico-degenerativas e/ou que estão em fase terminal, na perspectiva de promover conforto e bem-estar. Esse tipo de assistência é caracterizado não só pelos cuidados prestados ao paciente enfermo, cuja doença não responde mais ao tratamento curativo, mas também, aos seus familiares. ${ }^{10-12}$

Os cuidados paliativos têm como objetivo proporcionar uma melhor qualidade de vida, oferecendo condições que viabilizem e incentivem o paciente a viver o final de sua vida de forma produtiva, útil e que seja gratificante para o mesmo. Tais cuidados estão voltados à reabilitação física, mental e ao apoio profissional diante das limitações psicológicas, sociais e espirituais do paciente. Portanto, a assistência deve ser prestada por uma equipe multiprofissional de saúde, garantindo, desta forma, uma assistência integral e humanizada. ${ }^{10-12}$

A UTI tem sido palco da terminalidade humana nos dias atuais, decorrente dos avanços técnico-científicos que permeiam esta assistência. No entanto, diante de sua característica de resgatar vidas, há um indiscutível conflito entre a obstinação terapêutica e a prática dos cuidados paliativos neste cenário, principalmente pela dificuldade da equipe de profissionais intensivistas e dos familiares do paciente de reconhecer que este não tem mais perspectivas de cura e que agora precisa de cuidados que o possibilite ter uma morte digna, respeitosa e confortável. ${ }^{6}$

o conceito de "boa morte", no que diz respeito aos cuidados ao paciente terminal, tem sido utilizado quando estão presentes determinadas características, tais como: morte sem dor; morte ocorrendo com os desejos do paciente sendo respeitados (verbalizados ou registrados nas diretivas antecipadas); morte em casa cercado pelos familiares e amigos; ausência de evitável infortúnio e sofrimento para o paciente, sua família e o cuidador; morte em um contexto onde as necessidades do paciente estejam resolvidas e ocorrendo com uma boa relação entre o paciente e sua família com os profissionais de saúde. ${ }^{1}$

A ideia de realização de cuidados paliativos com qualidade, mesmo num cenário de grandes recursos tecnológicos como a UTI é relevante e necessária; assim, ao reconhecer que um paciente se encontra em fase terminal, o enfermeiro juntamente com a família do paciente e outros membros da equipe devem estabelecer contato precocemente para planejar cuidados que promovam atendimento integral das necessidades humanas. ${ }^{13}$

Analisando os discursos dos entrevistados foi possível perceber que a maioria apresenta uma compreensão fragilizada no que diz respeito aos cuidados paliativos. Em uma das citações o entrevistado (Enf.: 2) conceituou corretamente cuidados paliativos como sendo “... um conjunto de ações multiprofissionais que visam oferecer conforto ao paciente e seus familiares [...]", e citou "compressas frias para hipertermia" como exemplo de ação de cuidados paliativos. Outro entrevistado (Enf.: 4) referiu que cuidado paliativo é "Dar a assistência precisa ao paciente que tem doença crônica e dor... aquela definitivamente acentuada".

Vale ressaltar que parte dos participantes do estudo apresentou compreensão condizente com a essência dos cuidados paliativos, porém, podemos acrescentar que estes cuidados devem ser implementados em pacientes que recebem o diagnóstico de uma doença sem possibilidade terapêutica de cura, estando estes em fase terminal ou não. Desta forma, tais cuidados devem ser empregados logo que o paciente tome conhecimento de seu diagnóstico e não apenas diante da terminalidade ou presença de dores intensas, na perspectiva de promover uma melhoria na qualidade de vida relacionada à saúde do paciente e sua família o mais precoce possível. 


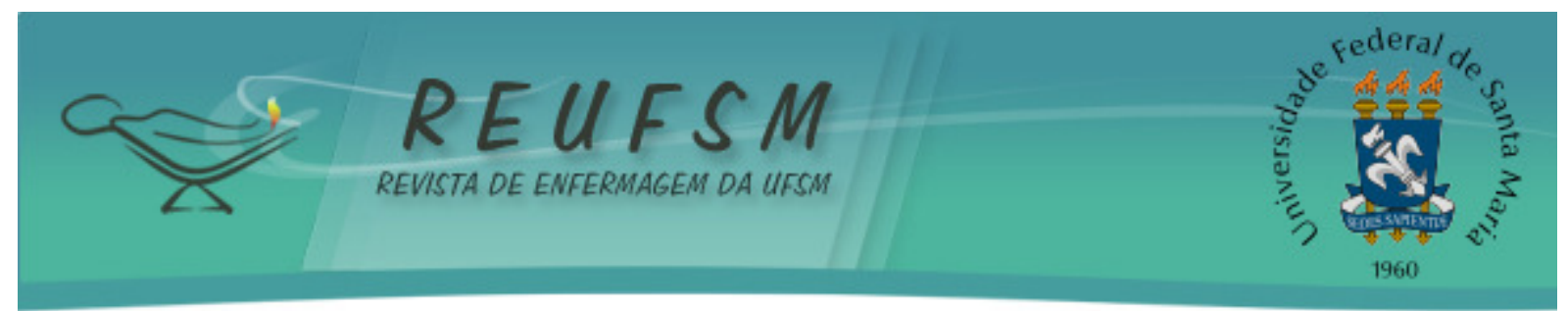

A ideia central (2) "Ações que não resultam em melhora", que emergiu dos discursos do sujeito coletivo, reflete a pouca aproximação que os entrevistados têm com os cuidados paliativos.

Dentre os discursos dos participantes do estudo, dois apresentaram um discurso que revela uma compreensão distante do contexto desta modalidade de cuidados: "São os primeiros atendimentos admissionais" (Enf.: 3 e 5). Este discurso revela um conhecimento fragilizado acerca da prática dos cuidados paliativos, pois estes, quando indicados, não são só realizados na admissão do paciente na instituição, mas, durante toda a permanência deste no serviço devendo ser empregados também ao núcleo familiar desde admissão até o acompanhamento à fase de luto.

A compreensão de um dos entrevistados sobre cuidados paliativos é que "São aqueles cuidados prestados ao paciente enfermo, mas que não resultará em melhora" (Enf.: 6); tal discurso pode ser atribuído ao significado que de fato cuidados paliativos não têm como premissa a cura, mas isso não implica dizer que não ofereça melhora do estado do paciente, uma vez que poderá proporcionar alívio de sintomas, maximização do conforto, dignidade, resultando em melhora da qualidade de vida em sua finitude.

Os cuidados paliativos na UTI, portanto, compreendem ações direcionadas aos pacientes críticos, em estágio terminal e sem possibilidades terapêuticas de cura. Para isso, deve haver consenso entre os membros da equipe e familiares do paciente quanto à manutenção de tratamentos fúteis, devendo priorizar cuidados que possam promover alívio de desconfortos físicos e psicológicos, para que o paciente tenha uma morte respeitosa, digna e tranquila, com inclusão da família nesta atenção. ${ }^{4}$

Dessa forma, os resultados revelam que a compreensão dos enfermeiros participantes do estudo traduz a sua formação profissional, na qual a abordagem sobre a morte, terminalidade e cuidados paliativos tem sido pouco expressiva, principalmente na atenção ao paciente crítico que se encontra num ambiente com tecnologia e recursos assistenciais de alta complexidade.

No tocante a questão "Que limites e possibilidades você encontra para realizar os cuidados paliativos?", emergiram dos discursos, as seguintes ideias centrais como representações dos limites para este cuidado: (1) ausência de profissionais preparados e de protocolo específico para cuidados paliativos; (2) apoio espiritual ao paciente. E como representação de possibilidades, emergiu a ideia: (3) realizar ações que proporcionem conforto e alívio da dor.

\begin{tabular}{|c|c|}
\hline Ideia central (1) & DSC (1) \\
\hline $\begin{array}{l}\text { Ausência de profissionais preparados } \\
\text { e de protocolo específico para } \\
\text { cuidados paliativos. }\end{array}$ & $\begin{array}{l}\text { [...] no momento meu local de trabalho não oferece esse } \\
\text { tipo de serviço. [...] muitas vezes não há equipe } \\
\text { profissional para prestar a assistência a esses pacientes } \\
\text { (Enf.: 1); [...] para realização de ações paliativas é } \\
\text { necessário que toda equipe multiprofissional se organize } \\
{[\ldots] \text { (Enf.: 2); Todas, pois não temos uma equipe }} \\
\text { multidisciplinar [...] (Enf.: 1). [...] prescrição médica } \\
\text { para analgésicos [...] (Enf.: 2). }\end{array}$ \\
\hline Idéia central (2) & DSC (2) \\
\hline Apoio espiritual ao paciente. & $\begin{array}{l}{[\ldots] \text { encontro mais limites na parte espiritual do paciente }} \\
{[\ldots] \text { (Enf.: 4); [...] não há participação efetiva e }} \\
\text { frequente de pastores, padres, etc.. [...] Enf.3 }\end{array}$ \\
\hline Idéia central (3) & DSC (3) \\
\hline $\begin{array}{l}\text { Realizar ações que proporcionem } \\
\text { conforto e alívio da dor. }\end{array}$ & $\begin{array}{l}{[\ldots] \text { proporcionar o alívio da dor, palavras de conforto }[\ldots]} \\
\text { (Enf.: } 1) ;[\ldots] \text { essas ações são de conforto }[\ldots] \text { (Enf.: } 3) \text {. }\end{array}$ \\
\hline
\end{tabular}

Figura 2- Ideia Central, DSC dos Enfermeiros em resposta a questão: Que limites e possibilidades você encontra para realizar os cuidados paliativos? Fonte: Dados da pesquisa, 2011. 


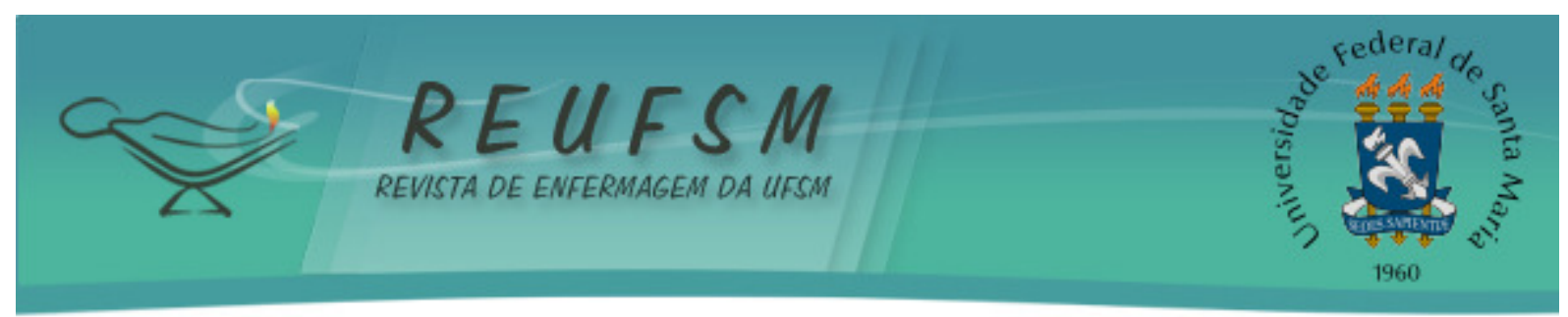

Com base nos discursos dos sujeitos coletivos apresentados na figura 2, destaca-se como ideia central (1) "Ausência de profissionais preparados e de protocolo específico para cuidados paliativos" representando as limitações enfrentadas pelos participantes do estudo para a realização de cuidados paliativos na unidade de terapia intensiva.

Ainda há grande número de instituições de saúde no Brasil que assistem pacientes críticos e/ou crônicos sem possibilidades terapêuticas de cura, que não integraram em sua prática os cuidados paliativos. Essa realidade está presente na fala de um dos participantes, quando afirma que "No momento meu local de trabalho não oferece esse tipo de serviço." (Enf.: 2).

O Brasil é um dos países que dispõe de poucas unidades de cuidados paliativos em seus hospitais e este tipo de cuidado passa a ser prestado de forma pontual, evidenciando a falta de integração dos cuidados paliativos e o sistema de saúde. ${ }^{14}$

Outro ponto abordado nos discursos refere-se ao fato de que ainda é escasso o número de equipes capacitadas para atuar frente aos pacientes em cuidados paliativos, um dos participantes do estudo afirmou que "Muitas vezes não há equipe profissional para prestar a assistência a esses pacientes." (Enf.:1). O cuidado paliativo requer planejamento interdisciplinar e ação multiprofissional, ou seja, se faz necessário uma equipe multiprofissional capacitada e especializada, para que se possa ofertar os cuidados paliativos de forma consistente e integral. ${ }^{11}$

Apesar dos cuidados paliativos terem sido eleitos pela OMS como uma prioridade há mais de uma década, a sua definição como área de atuação para diversas especialidades médicas brasileiras (geriatria, oncologia, clínica médica, pediatria, entre outros), tem sido pouco discutida. Consequentemente, tais profissionais sentem a falta desse treinamento, mantendo sua atuação no extremo da medicina curativa mesmo naqueles casos onde essa prática mostra-se ineficaz; por desconhecimento, ainda hoje, alguns médicos questionam o amparo ético e legal de prover cuidados paliativos e limitação de tratamento em pacientes em fase final de doença. ${ }^{15}$

Além da equipe médica, o cuidado paliativo envolve em sua abordagem a necessidade de uma equipe multidisciplinar, integrando várias especialidades já que este tipo de cuidado tem por objetivo identificar e dirimir os problemas relacionados à internação, na esfera física, psicológica, espiritual e/ou social do paciente em busca de melhor qualidade de vida. ${ }^{15}$

Deste modo a capacitação dos profissionais que atuam nas UTI's é imperativa, havendo necessidade de proporcionar a estes a educação continuada, especialmente no tocante aos cuidados paliativos, considerando que este é um tema pouco abordado e discutido na prática assistencial e na formação profissional. Com isso, os enfermeiros tornar-se-ão aptos para prestarem assistência com excelência em unidades de atendimento a pacientes críticos no ambiente hospitalar. ${ }^{16-17}$

Além da formação acadêmica para que o enfermeiro possa efetivamente pôr em prática as estratégias de cuidados paliativos se faz necessário que seu emocional esteja em equilíbrio, para assistir o paciente e sua família frente à terminalidade, realçando ainda a importância do mesmo como elo chave dentro da equipe paliativista. ${ }^{18}$

A necessidade de capacitação da equipe para atuar frente aos cuidados paliativos está realçada na fala de um dos enfermeiros participantes do estudo quando cita: "[...] se faz necessário uma prescrição médica" (Enf.: 2). Na realidade, para se desenvolver ações de cuidados paliativos deve existir a determinação de um conjunto de estratégias que compõe a modalidade deste cuidado, e não apenas uma prescrição médica isolada.

$\mathrm{Na}$ UTI, deve-se estabelecer como princípios fundamentais de cuidados paliativos a aceitação da morte de forma natural, priorizar a necessidade e interesse do paciente, não adotar condutas diagnósticas e terapêuticas fúteis, garantir a qualidade de vida e do morrer, aliviar a dor e/ou outros sintomas que causem desconforto físico, espiritual, psicológico, social do paciente e de seus familiares, respeitar a autonomia da paciente, 


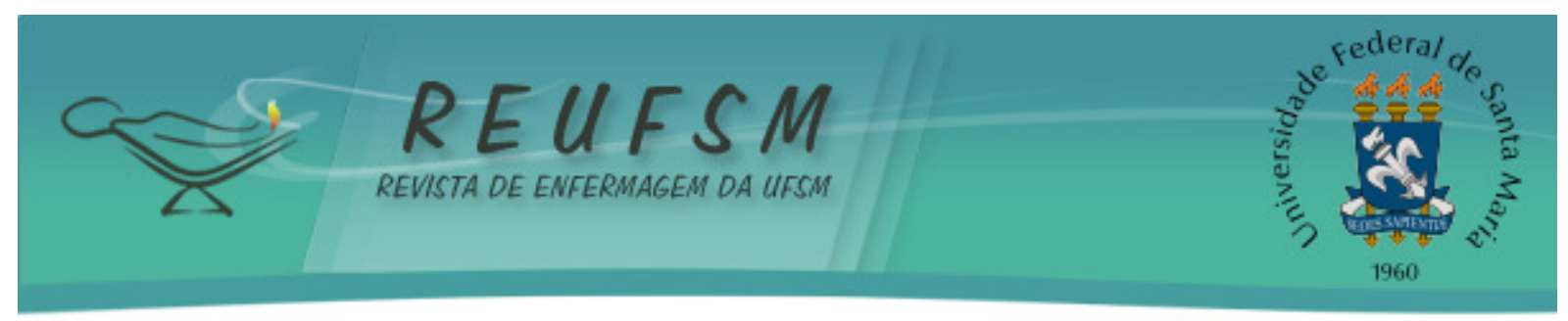

promover ações interdisciplinares, entre outros. As ações devem ser planejadas, envolvendo paciente, familiares e equipe de saúde e serem direcionadas para o estabelecimento de comunicação efetiva, ações que proporcionem maximização de conforto, bem-estar, com o máximo de beneficência possível e não-maleficência. ${ }^{4}$

$\mathrm{Na}$ perspectiva de promover a qualidade de vida do paciente, o enfermeiro deverá estar apto para exercer sua prática de forma autônoma, executando de forma sistematizada ações paliativas, por meio da aplicação do processo de enfermagem, identificando os diagnósticos e propondo intervenções de enfermagem.

A prática de enfermagem sistematizada favorece a identificação das necessidades de cuidado manifestadas e/ou referidas pelos clientes e familiares em sua totalidade, bem como a articulação e negociação com os demais membros da equipe de saúde em nome da concretização e melhorias do cuidado, constituindo uma estratégia adequada a uma prática centrada na pessoa e não apenas nas tarefas. ${ }^{19}$

A ideia central (2) "apoio espiritual ao paciente" que insurgiu dos discursos do sujeito coletivo, reflete a preocupação dos enfermeiros participantes com a necessidade espiritual do paciente, demonstrando uma visão que extrapola a esfera biológica do ser.

Neste ínterim, um dos participantes revelou que dentre as dificuldades encontradas no contexto dos cuidados paliativos destaca-se a atenção espiritual e logo justifica sua resposta revelando que cada paciente possui sua particularidade religiosa. Além disso, no cenário da terapia intensiva [...] não há participação efetiva e frequente de pastores, padres, etc.. [...] (Enf:.3), que possa atender adequadamente a esta necessidade.

Estudos têm revelado que mediante o incremento dos avanços tecnológicos na área da saúde, com prolongamento da vida de pacientes com doenças crônicas e degenerativas, tem se sobressaído a necessidade espiritual destes pacientes e familiares, buscando de forma mais intensa a religiosidade. ${ }^{14}$

Nesse contexto, surge um grande desafio para os profissionais de saúde, principalmente os que estão envolvidos numa assistência permeada de aparatos tecnológicos como na UTI, no tocante às questões sobre o equilíbrio entre as dimensões física, emocional e espiritual do ser humano, destacando-se a espiritualidade como fonte de aumento do bem-estar e da qualidade de vida, mediante aproximação da sua finitude. ${ }^{20}$ Evidentemente a necessidade espiritual do paciente criticamente enfermo e de seus familiares aflora com maior intensidade na iminência da morte, sendo pois cabível o seu atendimento e para isto os enfermeiros devem adquirir habilidade cognitiva para a identificação desta necessidade e prover medidas resolutivas.

A ideia central (3) "Realizar ações que proporcionem conforto e alívio da dor", denota possibilidades de ações paliativas a serem desenvolvidas na unidade de terapia intensiva, ressaltadas pelos participantes do estudo, no discurso do sujeito coletivo.

A literatura nacional e internacional aponta a dor como o $5^{\circ}$ sinal vital, devendo ser investigada sua presença com a mesma frequência que é realizada a monitorização dos outros sinais vitais: temperatura, frequência respiratória, frequência cardíaca e pressão arterial. É reconhecida como um sintoma de caráter multidimensional, incluindo dimensões neurofisiológica, psicossocial, cognitivo-cultural, comportamental e sensorial, cabendo ao enfermeiro adquirir habilidade para identificar, perceber, mensurar e avaliar a repercussão deste sintoma para o conforto do paciente e sua qualidade de vida relacionada à saúde. ${ }^{21}$

0 controle da dor é uma das principais metas do protocolo de cuidados paliativos, ressaltados pela OMS. ${ }^{22}$ No cenário da terapia intensiva, o controle dos sintomas de pacientes com diagnósticos de doenças sem possibilidades de cura, tem sido bastante discutido na literatura. Num estudo envolvendo 9105 pacientes atendidos em hospitais de ensino americanos, foi constatado que $50 \%$ destes pacientes que foram a óbito, revelaram nos últimos três dias de vida sentir dores, que variaram de média a grave intensidade. ${ }^{14}$ 


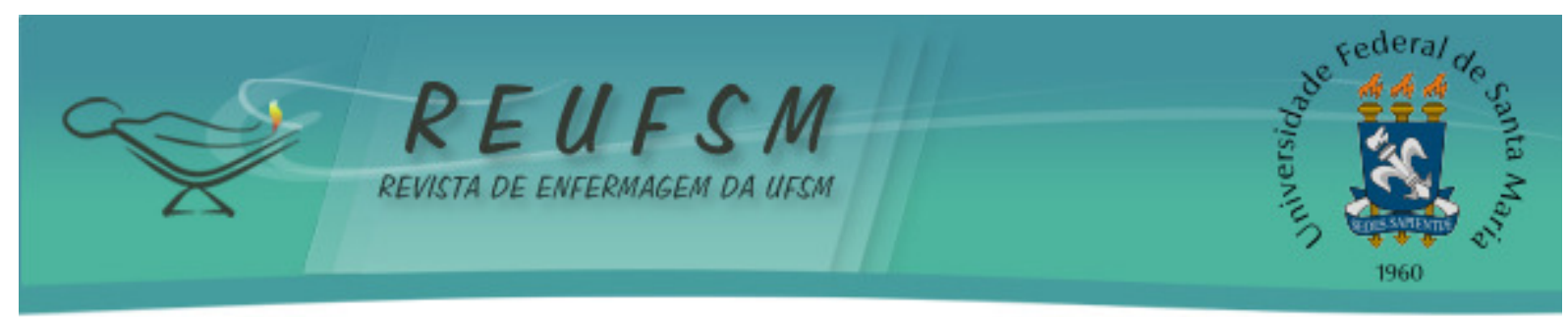

Considerando que a equipe de enfermagem assiste continuamente o paciente na UTI é de sua competência também identificar a presença de dor, avaliá-la utilizando escalas para mensurar a sua intensidade, notificar no prontuário e à equipe médica. Para o alívio da dor, o enfermeiro poderá instituir medidas não-farmacológicas e/ou administrar analgesia conforme protocolos e prescrição médica, de acordo com a situação clínica de cada paciente. ${ }^{17,23}$

Outras ações paliativas direcionadas a pacientes em estágio de terminalidade na UTI, como higiene, conforto, respeito, interação, comunicação, envolvimento da família neste cenário, entre outros, são possíveis de serem realizadas pelos enfermeiros intensivistas, mesmo que ainda não haja protocolos estabelecidos.

Portanto, corroborando com o discurso dos enfermeiros participantes do estudo, quando reconhecem o conforto e alívio da dor como estratégias paliativas possíveis de serem desenvolvidas dentro da UTI, torna-se imprescindível que a equipe de enfermagem esteja capacitada a promover conforto físico, psicológico e espiritual ao paciente em todas as suas dimensões. Desse modo, as ações paliativas em detrimento da complexidade tecnológica da unidade, podem permitir que este paciente que está em sua finitude, complete seu ciclo vital com dignidade, respeito e com o mínimo de sofrimento possível.

\section{CONSIDERAÇÕES FINAIS}

Essa pesquisa teceu discussões a respeito dos cuidados paliativos em UTI com enfoque na compreensão, nos limites e possibilidades enfrentados por profissionais de enfermagem, no tocante à execução dos cuidados paliativos na UTI.

Com este estudo foi possível observar que os cuidados paliativos são reconhecidos como uma importante questão de Saúde Pública, pois envolve o sofrimento, a dignidade, o cuidado das necessidades humanas e qualidade de vida das pessoas afetadas por uma doença crônica e degenerativa ou que está em fase final de vida. Com isso a preocupação deve ser não só com o paciente que está sob nossos cuidados, mas também com seus familiares.

Os discursos individuais dos participantes desse estudo revelaram ideias pouco consistentes e confusas em relação ao que eles entendem por cuidados paliativos, alguns, até, fugindo totalmente da filosofia do atendimento. Em outro momento percebe-se a dificuldade dos entrevistados em citar quais as ações paliativas realizadas por eles são mais comuns ou utilizadas em pacientes terminais.

No que tange aos limites e possibilidades para implementação desses cuidados, revelaram como limites o apoio espiritual, falta de profissionais, não oferta de cuidados paliativos pela equipe, já em relação às possibilidades ressaltaram a organização da equipe para planejar e definir a oferta desses cuidados.

Para realizar cuidados de qualidade no intuito de responder a situações complexas e necessidades de um doente terminal e sua família, é essencial que os profissionais façam parte de equipes especializada em cuidados paliativos e tenham uma formação específica para isso, pois o enfermeiro que possui apenas a graduação pode enfrentar dificuldades diante de pacientes fora de possibilidades terapêuticas de cura.

Para tanto, uma das medidas, para melhoria da formação do enfermeiro, é a inclusão de disciplinas que discutam sobre cuidados prestados à pacientes fora da possibilidade terapêutica, abordando temas como luto e o fim da vida, proporcionado aos graduandos uma significativa compreensão dos processos da morte. Para que esses futuros profissionais ofereçam um atendimento individualizado, priorizando a dignidade humana.

Ressaltamos que este estudo apresenta algumas limitações no tocante à análise e discussões dos resultados, decorrentes da fragilidade dos discursos dos entrevistados, que revelou um conhecimento fragmentado sobre cuidados paliativos, não sendo possível apreender uma relação apropriada sobre estes cuidados no contexto da terapia intensiva. Isto 


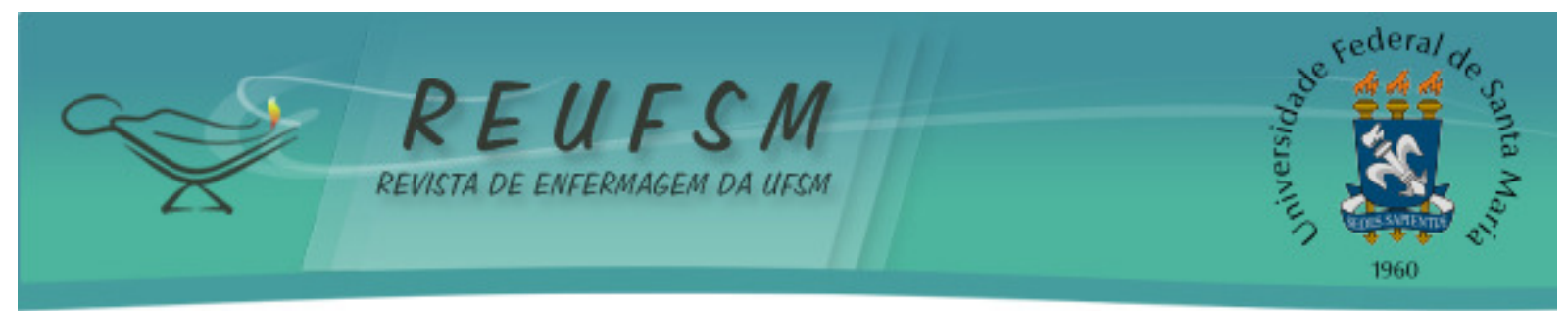

nos faz pensar que talvez não seja um problema isolado, de uma instituição apenas, mas que seja a realidade de muitas instituições no nosso país, resultante de uma formação acadêmica voltada especificamente para o tecnicismo, sem considerar as diversas dimensões do ser humano no seu processo vital, repercutindo diretamente na conduta do profissional de saúde.

Assim, torna-se perceptível que esses profissionais necessitam integralizar novos conceitos sobre cuidados paliativos, para que se possa ter uma melhor compreensão acerca desses cuidados em qualquer área de atenção ao ser humano. Desta forma, se faz necessário novos estudos, intervenções e treinamentos para os profissionais, buscando assim, uma melhoria no sistema desses cuidados.

\section{REFERËNCIAS}

1. Floriani CA, Schramm FR. Desafios morais e operacionais da inclusão dos cuidados paliativos na rede de atenção básica. Cad Saúde Pública [internet]. 2007 [acesso em 2012 abr 19];23(9):2072-80. Disponível em: http://www.scielo.br/pdf/csp/v23n9/08.pdf

2. Ministério da Saúde (BR). Instituto Nacional de Câncer-INCA. Ações de enfermagem para o controle do câncer: uma proposta de integração ensino-serviço. $3^{\mathrm{a}}$ ed. Rio de Janeiro (RJ): 2008.

3. Costa Filho RC, Costa JLF, Gutierrez LFBR, Mesquita AF. Como Implementar Cuidados Paliativos de Qualidade na Unidade de Terapia Intensiva. Rev Bras Ter Intensiva [internet]. 2008 [acesso em $2012 \mathrm{abr}$ 19];20(1):88-92. Disponível em:

http://www.scielo.br/pdf/rbti/v20n1/a14v20n1.pdf

4. Moritz RD, Lago PM, Souza RP, Silva NB, Meneses FA, Othero JCB et al. Terminalidade e cuidados na unidade de terapia intensiva. Rev Bras Ter Intensiva [internet]. 2008 [acesso em 2012 Abr 19];20(4):422-28. Disponível em:

http://www.scielo.br/pdf/rbti/v20n4/v20n4a16.pdf

5. Melo AGC. Os cuidados paliativos no Brasil. Rev Brasileira de Cuidados Paliativos [internet]. 2008 [acessado em 2012 Abr 19];1(1):5-8. Disponível em:

http://www.cuidadospaliativos.com.br/img/din/file/RBCP1.pdf

6. Menegócio AM, Larissa R, Silva SR. Cuidados paliativos em unidade de terapia intensiva: quando iniciá-los. Anuário da Produção Acadêmica Docente. 2010;4(7):163-74.

7. Polit DF, Beck CT. Fundamentos de pesquisa em enfermagem. Avaliação de evidências para a prática da enfermagem. $7^{\mathrm{a}}$ ed. Porto Alegre (RS); 2011.

8. Lefèvre $F$, Lefèvre AMC. O sujeito coletivo que fala. Interface (Botucatu) [internet]. 2006 [acesso em 2012 Abr 12];10(20):517-24. Disponível em:

http://www.scielo.br/pdf/icse/v10n20/17.pdf

9. Brasil. Conselho Nacional de Saúde. Diretrizes e normas regulamentadoras de pesquisa envolvendo seres humanos. Resolução n 196, de 10 de outubro de 1996.

10. Pessini L, Bertachini L. Novas perspectivas em cuidados paliativos. Acta Bioethica. 2006;12(2):231-42.

11. Remedi PP, Mello DF, Menossi MJ, Lima RAG. Cuidados paliativos para adolescentes com câncer: uma revisão de literatura. Rev Bras Enferm. 2009;62(1):107-12.

12. Silva EP, Sudigursky D. Concepções sobre cuidados paliativos: revisão bibliográfica. Acta Paul Enferm. 2008;21(3):504-08. 


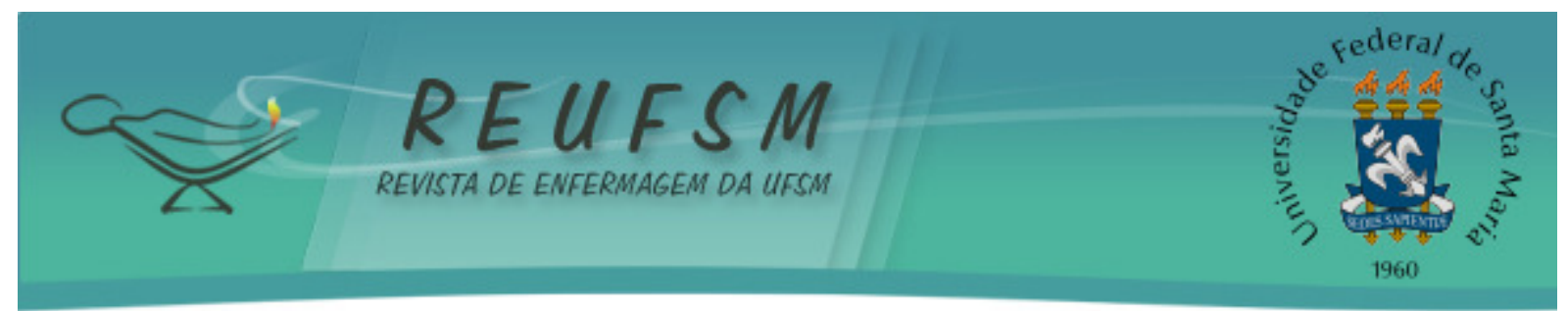

13. Fonseca JVC, Rebelo T. Necessidades de cuidados de enfermagem do cuidador da pessoa sob cuidados paliativos. Rev Bras Enferm [internet]. 2011 [acesso em $2012 \mathrm{Abr}$ 12];24(1):180-4. Disponível em: http://www.scielo.br/pdf/reben/v64n1/v64n1a26.pdf

14. Costa Filho RC, Costa JLF, Gutierrez FLBR, Mesquita AF. Como Implementar Cuidados Paliativos de Qualidade na Unidade de Terapia Intensiva. Rev Bras Ter Intensiva [internet]. 2008 [acesso em 2012 Dez 04];20(1):88-92. Disponível em:

http://www.scielo.br/pdf/rbti/v20n1/a14v20n1.pdf

15. Piva JP, Garcia PCR, Lago PM. Dilemas e dificuldades envolvendo decisões de final de vida e oferta de cuidados paliativos em pediatria. Rev Bras Ter Intensiva [internet]. 2011 [acesso em $2012 \mathrm{Abr}$ 10];23(1):78-86. Disponível em: http://www.scielo.br/pdf/rbti/v23n1/a13v23n1.pdf

16. Lazzari DD, Schmidt N, Jung W. Educação continuada em unidade de terapia intensiva na percepção de Enfermeiras. Rev Enferm UFSM [internet]. 2012 [acesso em 2012 Jul 14];2(1):88-96. Disponível em: http://cascavel.ufsm.br/revistas/ojs-

2.2.2/index.php/reufsm/article/download/4592/3130

17. Oliveira SG, Quintana AM, Budó MLD, Bertolino KCO, Kruse MHL. A formação do enfermeiro frente às necessidades emergentes da terminalidade do indivíduo. Rev Enferm UFSM [internet]. 2011 [acesso em 2012 Dez 04];1(1):97-102. Disponível em: http://cascavel.ufsm.br/revistas/ojs-2.2.2/index.php/reufsm/article/download/1996/1519

18. Fonseca JVC, Rebelo T. Necessidades de cuidados de enfermagem do cuidador da pessoa sob cuidados paliativos. Rev Bras Enferm [internet]. 2011 [acesso em 2012 Dez 04];24(1):180-4. Disponível em: http://www.scielo.br/pdf/reben/v64n1/v64n1a26.pdf

19. Silva MM, Moreira MC. Sistematização da assistência de enfermagem em cuidados paliativos na oncologia: visão dos enfermeiros. Acta Paul Enferm [internet]. 2011 [acesso em 2012 Abr 10];24(2):172-8. Disponível em: http://www.scielo.br/pdf/ape/v24n2/03.pdf

20. Wachholtz $A B$, Keefe FJ. What physicians should know about spirituality and chronic pain. South Med J. 2006;99(10):1174-5.

21. Bottega FH, Fontana RT. A dor como quinto sinal vital: utilização da escala de avaliação por enfermeiros de um hospital geral. Texto \& Contexto Enferm [internet]. 2010 [acesso em 2012 Dez 04];19(2):283-90. Disponível em: http://www.scielo.br/pdf/tce/v19n2/09.pdf

22. Salamonde GLF, Vercosa N, Barrucand L, Costa AFC. Análise clínica e terapêutica dos pacientes oncológicos atendidos no programa de dor e cuidados paliativos do Hospital Universitário Clementino Fraga Filho no ano de 2003. Rev Bras Anestesiol. 2006;56(6):602-18.

23. Fontes $\mathrm{KB}$, Jaques $\mathrm{AE}$. $\mathrm{O}$ papel da enfermagem frente ao monitoramento da dor como $5^{\circ}$ sinal vital. Cienc Cuid Saude. 2007;6 Supl 2:481-7.

Data de recebimento: $15 / 07 / 2012$

Data de aceite: 02/01/2013

Contato com autor responsável: Cecília Danielle Bezerra Oliveira

Universidade Estadual da Paraíba

Endereço: Rua Baraúnas, 351 - Bairro Universitário - Campina Grande-PB, CEP 58429-500

E-mail: cecilia.dani@hotmail.com 